\title{
El profesorado y la responsabilidad social universitaria: un análisis cualitativo de redes
}

\author{
Gustavo A. Rubio-Rodríguez ${ }^{1}$ y Alexander Blandón-López ${ }^{2}$ \\ (1) Programa de Contaduría pública, Corporación Universitaria Minuto de Dios-Uniminuto, Calle 87 No. 20-98, Ibagué, \\ Colombia (correo-e: gustavo.rubio-r@uniminuto.edu.co). \\ (2) Facultad Ciencias Económicas y Administrativas, Universidad del Tolima, Calle 42 \#1B-1 Barrio Santa Helena Parte \\ Alta Ibagué, Colombia (correo-e: ablandonl@ut.edu.co).
}

Recibido Jul. 14, 2020; Aceptado Sep. 10, 2020; Versión final Nov. 10, 2020, Publicado Feb. 2021

\begin{abstract}
Resumen
Este estudio tiene por objeto analizar el concepto de responsabilidad social universitaria (RSU) emitido por un colectivo profesoral en una institución de educación superior colombiana, con la intención de optimizar sus prácticas. La muestra correspondió a 54 sujetos, seleccionados a través de muestreo por afijación óptima, y pertenecientes a diferentes programas académicos. Mediante un análisis cualitativo de redes, se logró determinar las percepciones predominantes que manifiestan los profesores en RSU. Como hallazgo representativo, los profesores asumen una conducta moralista, con un marcado adeudo consigo mismo, y un estricto sentido de pertenecía para con la sociedad. La conclusión más significativa, reside en que la participación del profesorado es vital para posibilitar mejores condiciones de vida del entorno, dado que su comportamiento determina las prácticas que las universidades deben adelantar en RSU. Así, se debe apelar a un modelo de gobernanza reticular y no a modelos de estructura vertical que prevalecen en algunas instituciones de educación superior.
\end{abstract}

Palabras clave: responsabilidad social universitaria; educación superior; profesores; percepciones; programas académicos

\section{Professors and university social responsibility: a qualitative network analysis}

\begin{abstract}
The objective of this research study is to examine and to optimize the application of the university social responsibility (USR) concept issued by professors at a Colombian higher education institution. The sample (54 subjects) is selected by optimal allocation from different academic programs. The results obtained by applying a network qualitative analysis show the most predominant perceptions among professors about USR. Professors assume a moralistic behavior. They feel that they are in debt with themselves and that they belong to society. It is concluded that professor engagement is vital to improve living conditions on campus environments since their behaviors determine the USR practices that universities should implement. This suggests that higher education institutions should appeal to a reticular governance model and not to a vertical structure model, which prevails in a number of higher education institutions.
\end{abstract}




\section{INTRODUCCIÓN}

Para hablar de RSU, primeramente se debe indicar que este tipo de responsabilidad tiene su génesis en la responsabilidad social empresarial, y ésta surge de la necesidad de ser implementada en el escenario organizacional (Rubio-Rodríguez et al. 2020). La responsabilidad social nace en el siglo XX, alrededor del año 1920, y toma fuerza en 1970, y se origina como una iniciativa para responder de manera ética al uso de recursos naturales y sociales (Rodríguez, 2017). También, es vinculante mencionar, que nace como producto de diferentes sucesos a los cuales la humanidad tuvo que enfrentarse a comienzos del siglo XXI, tales como el calentamiento global, la hambruna, la evolución de la tecnología, entre otros, induciendo a tomar medidas para el desarrollo de una sociedad íntegra en busca de promover la participación de la ciudadanía, que trabaje en armonía para responder a los desafíos que hoy nos enfrentamos tanto locales como globales, procurando la conservación del medio ambiente (Larrán y Andrades, 2017).

Asimismo, la responsabilidad social se perfecciona en las organizaciones cuando éstas aceptan el papel que juegan en su entorno, generando conciencia bajo una conducta altruista; es decir, dejar de pensar solo en necesidades individuales y empezar a preocuparse por los problemas que poseen aquellos que hacen parte del medio que los rodea (Ayala-Rodríguez et al. 2019). De igual forma, la responsabilidad social expresa la relación que hay entre los individuos, su entorno y sus principios éticos, y trabaja en armonía para contribuir por un mejor futuro en conjunto con otras personas, de tal manera que éstas logren apropiarse de verdaderas prácticas sociales (Vasilescu et al. 2010). La responsabilidad social empresarial no ha sido objeto de ser implementada exclusivamente por el sector empresarial, sino, que en la actualidad las instituciones de educación superior han optado por incluir la RSU en el desarrollo de sus actividades y objeto social, para responder de manera eficiente a los desafíos que afronta asiduamente (Appe et al. 2017).

Ahora bien, una de las razones por las cuales las universidades se someten a procesos de calidad, rendición de cuentas, necesidad de trasferencia, etc., radica en que las funciones sustantivas que emprende, deben evidenciar acciones al servicio de la sociedad, y que la motivación a dicho sometimiento no se concentra en intereses netamente institucionales (Vallaeys, 2020; Pedraja-Rejas et al. 2012). De allí, que no es menester de este tipo de organizaciones demostrar ante las autoridades competentes en materia educativa, altos estándares académicos, o la figuración en el ranking de las mejores universidades por la alta producción intelectual que generan, cuando no están aportando una mejora de la calidad de vida de una colectividad menos favorecida. Por tanto, las universidades deben tomar conciencia del papel que ejercen dentro de sí misma y del entorno.

De otro lado, en cuanto a la puesta en marcha de políticas de responsabilidad relacionadas con la mejora del clima organizacional, rendimientos, crecimiento, sostenibilidad, etc., cabe destacar el trabajo presentado por Vallaeys y Álvarez (2019:1), en el cual manifiesta, que "la responsabilidad social universitaria es una nueva política de gestión universitaria que se va desarrollando en Latinoamérica para responder a los impactos organizacionales y académicos de la universidad". Dichos impactos están representados, connaturalmente, en acciones que propugnan por mejorar la relación que se forja entre los diferentes agentes de interés que participan en el escenario universitario. Así lo ratifica la investigación adelantada por Flórez et al. (2017), al acotar que el comportamiento de las organizaciones educativas tiene su esencia en las "transacciones respetuosas que concibe una organización con su entorno y todos los agentes de interés que la componen" (p. 85).

Asimismo, según Urquijo et al. (2019), cuando se habla de políticas de responsabilidad social, se especula en la correspondencia que se promueve entre la empresa, el medio ambiente y el contexto social. Exiguamente se cavila en el recurso humano, y este aspecto se ha delegado en áreas como la gestión humana. Sin embargo, vale la pena señalar que la responsabilidad social debe ser concebida como una estrategia transversal, y como una herramienta intangible invaluable que asienta al ser humano enriqueciendo su rol, contribuye a mejorar la calidad de vida de los colaboradores y la relación de las empresas con su entorno inmediato. Así lo interpretan Turker (2009:85) en su trabajo investigativo "las nuevas políticas que implementen las empresas deben estar encaminadas en garantizar una mayor calidad de vida laboral y familiar a sus trabajadores, implementando nuevas formas de trabajar como programas sociales de ayuda a familiares de los empleados".

Cerrando este acápite, las políticas que estén o vayan a ser implementadas por las organizaciones educativas de orden superior, según Zavala-Guirado et al. (2018), les incumbe estar en incesante cambio, y más en estos tiempos cuando se observan tendencias globales que pueden transformar de una manera contundente el escenario universitario, enfrentando a estas instituciones a retos de adaptación a esos nuevos escenarios, razón por la cual las organizaciones de educación superior, en la actualidad, se encuentran en la búsqueda de fortalecimiento en sus modelos educativos, mediante esquemas de competencia relacionados con la responsabilidad social, que impacten esencialmente los agentes de interés que de éstas forman parte. 
El objetivo de este manuscrito consiste en analizar el concepto de RSU que arroga el cuerpo profesoral de una institución universitaria de la Ciudad de Ibagué, Colombia, con el propósito de perfeccionar su conducta en esta materia. Para ello, es necesario precisar que las políticas de responsabilidad social emanadas de la institución educativa, se deben enmarcar en aquellas acciones que optimizan la labor, fortalecen el vínculo y potencializan el compromiso de los grupos de interés, en el intento por minimizar las relaciones establecidas entre uno y otro, generando mayor sensibilidad sobre la importancia que representa el recurso humano para estas instituciones.

Por otra parte, cabe señalar que el estudio destaca primordialmente aspectos demográficos como "edad y nivel educativo", que alcanzaban los profesores al momento de ser encuestados, los cuales se tomaron como variables. Potencialmente al relacionar estas variables, el concepto de RSU emitido por los profesores puede residir en una posición moralista en cuanto su comportamiento, con un marcado compromiso consigo mismo y con la sociedad, pero también interesado en vincular diferentes grupos sociales en los procesos de formación. Dicha posición, que puede presentarse como extraña a la luz de la conducta que axiológicamente guardar el corazón del ser humano, puede involucrar otros deseos que conducen a generar efectos contrarios.

\section{OTROS ANTECEDENTES: EL PROFESORADO}

El profesorado juega un papel protagónico para la RSU, en razón a que en representación de la institución de educación transfiere el conocimiento a nuevas generaciones, enseñando, formando e investigando (Fonseca et al. 2019). En consecuencia, al desarrollar tales actividades, las organizaciones educativas estarían descubriendo la posibilidad de trasladar ese conocimiento más allá del aula a sus propias operaciones institucionales (Jie y Huam, 2019). Esto significa profundizar su compromiso con la responsabilidad social a nivel académico, principalmente a través de acciones que redunden para beneficio de los entornos. Por tanto, para una institución universitaria es imperativo garantizar las capacidades de los recursos internos relacionados a los servicios educativos que se brindan, con miras a alcanzar la calidad en la gestión universitaria (Montalvo et al. 2018).

Otro de los grandes aportes que puede generar el profesorado a la RSU, radica en la forma de comportarse con sus homólogos. Empero, esta conducta depende de la relación que la institución educativa haya alcanzado con sus grupos de interés, dado que estos afectan y son afectados por las acciones de la organización. Por tanto, las universidades como cualquier entidad indistintamente de su naturaleza, deben velar por el bienestar de sus miembros, y brindar un ambiente físico y humano apto para laboral. Un estudio adelantado por Obeidat (2016:365), así lo corrobora, al concluir que "la responsabilidad social empresarial es el predictor más fuerte del compromiso de los empleados. Esto es apoyado por Vallaeys (2020), quien indicó que la forma en que se trata a los empleados es la prueba definitiva de responsabilidad social de una empresa.

En concordancia con lo anterior, las universidades a través de su cuerpo profesoral tienen la oportunidad de priorizar el trabajo con los sectores más desfavorecidos, mediante la formulación y ejecución de proyectos sociales. Baste lo anterior para señalar esta realidad que despliega la universidad, que sin lugar a dudas posibilita optimizar los procesos externos. Estos resultados concuerdan con el trabajo de Santos et al. (2020), quienes consideran que las instituciones de educación superior tienen un gran potencial en términos de contribuciones para resolver problemas sociales, mejorar el crecimiento y el desarrollo económico, y fomentar el respeto y la protección del medio ambiente. En la misma línea, Beltrán-Llevador et al. (2014), aseveran que los educadores son llamados a convertirse en agentes de servicio comunitario comprometidos con la sociedad civil.

En cuanto a los estudios concernientes al profesorado y la RSU, se destaca el adelantado por Martí-Noguera et al. (2018), en el que alude que los profesores cumplen un papel de agente de transformación en la sociedad. Por tanto, en palabras de Ramos-Monge et al. (2019), la planta profesoral en esencia tiene un gran impacto social, pero son todos los miembros de las instituciones de educación superior quienes decididamente deben integrar el concepto de responsabilidad social en las áreas sustantivas, para que esta acción contribuya a su consolidación en el resto de la sociedad, evitando señalamientos de cualquier índole en su contra. Del mismo modo Ayala-Rodríguez et al. (2019), afirman que es esencial que la universidad establezca agendas de cooperación interna y externa, facilitando así que alcancen un impacto apropiado en el contexto social.

De otra parte, las instituciones universitarias manifiestan una postura transformadora en sus discursos, su aplicación presenta algunas carencias o limitaciones; para nombrar alguna: existen múltiples definiciones, enfoques e interpretaciones sobre el significado de la RSU que dificultan conseguir una actuación socialmente responsable que sea admitida en común acuerdo. En consecuencia, ciertas universidades se limitan a enseñar solo proyección social, y suponen un alto grado de sinonimia con RSU, ignorando su verdadera acepción (Vallaeys, 2018). Otros centros universitarios proporcionan un entorno de sensibilización que permite el uso y la transferencia de conocimiento para favorecer el entorno externo, desconociendo las 
parvedades que arroga el entorno interno (Duque y Cervantes-Cervantes, 2019). En definitiva, tanto la proyección social asociada a la RSU como el entorno interno, son aspectos sobre los cuales se debe seguir investigando.

\section{METODOLOGÍA}

El estudio comprende un análisis descriptivo. Desde lo descriptivo "se reseñan las características o rasgos de la situación o fenómeno objeto de estudio" (Bernal, 2016, p. 143). Al mismo tiempo, permite identificar aspectos importantes de un análisis descriptivo como la capacidad para seleccionar las características fundamentales de la población objeto de estudio y su descripción detallada. La estadística descriptiva tiene como objetivo describir el colectivo estudiado; este puede ser toda la población (censo) o una parte de la misma (muestra). Cuando se selecciona de una población adecuadamente un grupo de individuos a través de la muestra, la caracterización de éstos es considerada como el objetivo de la estadística descriptiva.

Para responder a los objetivos de la investigación, inicialmente se tomó una unidad muestral emanada del total de la población profesoral, la cual corresponde a 90 profesores. Respecto a la temporalidad del estudio, se llevó a cabo entre el año 2019 y 2020 en el marco de la tesis doctoral en ciencias sociales y de la educación adelantada en la Universidad de Huelva por uno de los autores. Asimismo, el trabajo de campo se adelantó con el diseño y la administración de un instrumento para medir las diferentes percepciones respecto el tema objeto de estudio, para luego analizarlas.

En cuanto a la universidad analizada, ésta corresponde a la Universidad Cooperativa de Colombia, la cual se encuentra ubicada en la ciudad de lbagué, Colombia. Esta institución educativa, se ha ido consolidando como una de las organizaciones de educación superior más importantes de la región. Amparada en su estructura multicampus, que es la extensión de la universidad en todo el país, cuenta con presencia en regiones apartadas de difícil acceso para brindar el servicio de educación. Su accionar académico y de gestión organizacional, ha sido reconocido por diferentes entes y gobiernos municipales y departamentales en donde opera, dada su vocación de servicio e interés por incluir socialmente al menos favorecido.

\section{Unidad muestral}

En la investigación se tomó como población objeto de estudio a los profesores de pregrado presencial, que conforman la planta del personal docente de la Universidad Cooperativa de Colombia. Los profesores seleccionados (54 sujetos) pertenecen a los programas de administración de empresas, contaduría pública, derecho, ingeniería civil, y medicina veterinaria y zootecnia.

La selección de los profesores se adelantó a través del muestreo aleatorio estratificado por afijación óptima. A partir de la demostración matemática relacionada en la Tabla 1, se calculó la muestra para la presente investigación la cual correspondió a 54 sujetos. Asimismo, en dicha tabla se evidencia el número de profesores que participaron por programa académico y su representación porcentual.

Tabla 1: Selección de la muestra por afijación óptima

\begin{tabular}{|c|c|c|c|c|}
\hline $\begin{array}{c}\text { Población } \\
\text { objeto de } \\
\text { estudio }\end{array}$ & Programas & $\begin{array}{c}\text { Número de } \\
\text { profesores } \\
N_{i}\end{array}$ & $\begin{array}{c}\text { Participación } \\
\text { por programa } \\
\hat{Y}(\%)\end{array}$ & $\begin{array}{c}n_{i}=\frac{\sqrt{a_{I}}}{\sqrt{\lambda}} \\
=\frac{n \sqrt{a_{I}}}{\sum_{k=1}^{L} \sqrt{a_{k}}}\end{array}$ \\
\hline \multirow{4}{*}{ Profesores } & $\begin{array}{c}\text { Administración de } \\
\text { Empresas }\end{array}$ & 7 & 8 & 9 \\
\cline { 2 - 5 } & Contaduría Publica & 17 & 19 & 13 \\
\cline { 2 - 5 } & Derecho & 24 & 27 & 12 \\
\cline { 2 - 5 } & Ingeniería Civil & 23 & 26 & 11 \\
\cline { 2 - 5 } & $\begin{array}{c}\text { Ingeniería de } \\
\text { Sistemas }\end{array}$ & 4 & 4 & 2 \\
\cline { 2 - 5 } & $\begin{array}{c}\text { Med. Veterinaria } \\
\text { Zootecnia }\end{array}$ & 13 & 14 & 2 \\
\cline { 2 - 5 } & Open Lingua & 2 & 2 & 54 \\
\hline Total & & 90 & 100 & 5 \\
\hline
\end{tabular}




\section{Técnica de recolección de información}

Para el estudio se empleó la encuesta, la cual recopiló información con fines de analizar las políticas, acciones o actividades que, en materia de RSU, se llevan a cabo en la universidad objeto de estudio. De igual forma, la información recolectada permitió comprender el compromiso que los profesores tienen frente al concepto de RSU. El instrumento tiene como finalidad ser administrado sin distinción de semestre académico, o de programa, puesto que las políticas de responsabilidad social deben ser aplicadas de forma transversal en la institución y a todos sus actores.

En cuanto a la estructura de la encuesta se decidió presentarla en dos dimensiones sobre las que giró el comportamiento de la misma: 1) caracterización del profesorado; y 2) concepto de RSU. Cada una de estas dimensiones mostraron un número suficiente de variables las cuales permitieron su medición. En este orden, las variables para la primera dimensión se midieron a través de la escala Likert con el propósito de evaluar aspectos demográficos de los encuestados. Así entonces, la escala Likert presentó seis puntos como opciones de repuesta, donde 1. totalmente de acuerdo, 2. de acuerdo, 3. indeciso, 4. en desacuerdo, 5. totalmente en desacuerdo, 6 . NS/NR. Se presentó un número impar en razón a que naturalmente la tendencia conlleva ubicar un término medio. La segunda dimensión se midió a través de una pregunta abierta en la que indagó acerca del concepto de RSU que les asiste a los profesores de la universidad estudiada.

\section{Tratamiento de la información}

El desarrollo de esta investigación empleó el Análisis Exploratorio de Datos (AED), el cual tiene como propósito según Batanero et al. (1991, p. 91): "En primer lugar, se disminuye la importancia visual de la representación de los datos, dándosela exclusivamente a los cálculos, y en segundo, se equipará el análisis con el modelo confirmatorio". En este tipo de análisis, el conjunto de valores de las variables observadas se supone que se ajusta a un modelo preestablecido, calculando los estadísticos para aceptar o no una hipótesis.

Una vez organizados los datos, el segundo paso de un AED, consiste en realizar un análisis estadístico gráfico y numérico de las variables del problema, con el fin de tener una idea inicial de la información contenida en el conjunto de datos, así como detectar la existencia de posibles errores en la modificación de los mismos. Los usuarios de las técnicas descriptivas clásicas saben que en éstas priman los índices de tendencia central y de dispersión, considerándose como secundarios los de forma y derivados gráficos que pueden obtenerse.

Asimismo, esta investigación descansa en el análisis de preguntas abiertas gestadas por las percepciones de los profesores abordados en el estudio. La forma como se procesó este tipo de información fue mediante la técnica de análisis por redes, que es de orden cualitativo hermenéutico con enfoque descriptivo. Según Thomas y Harden (2008), el metanálisis cualitativo incluye una secuencia de tres fases, las cuales fueron empleadas en este estudio: 1) Codificación abierta de la sección de texto del artículo: El proceso de codificación abierta se organiza de acuerdo con los estándares de análisis de contenido enumerados en la representación del texto (en este caso, la forma escrita). 2) Descripción de temas que permitan correlacionar las subdivisiones para generar categorías de análisis. 3) Utilización del software ATLAS.ti para obtener una nueva síntesis temática a partir de la propuesta gráfica de la red semántica.

\section{RESULTADOS}

Las variables a tener en cuenta para este primer análisis exploratorio de datos para los profesores, los presenta la Tabla 2.

Tabla 2: Variables de interés. Aspectos demográficos de los profesores

\begin{tabular}{|c|c|l|}
\hline Modulo & Variables & Significado \\
\hline \multirow{4}{*}{ A } & PD1 & Programa académico \\
\cline { 2 - 3 } & PD2 & Edad \\
\cline { 2 - 3 } & PD3 & Nivel educativo máximo \\
\hline
\end{tabular}

Contrastando la relación del número de estudiantes por número de profesores, se logra apreciar en la figura 1, que efectivamente los programas académicos que agrupan la mayoría de estudiantes de la universidad, concentra también el mayor número de profesores; de esta manera, los programas académicos que mayor participación presentan durante el proceso investigativo, fueron los programas de Contaduría Pública (24\%), Derecho (22\%) e Ingeniería Civil, juntos con una participación del $20 \%$. 


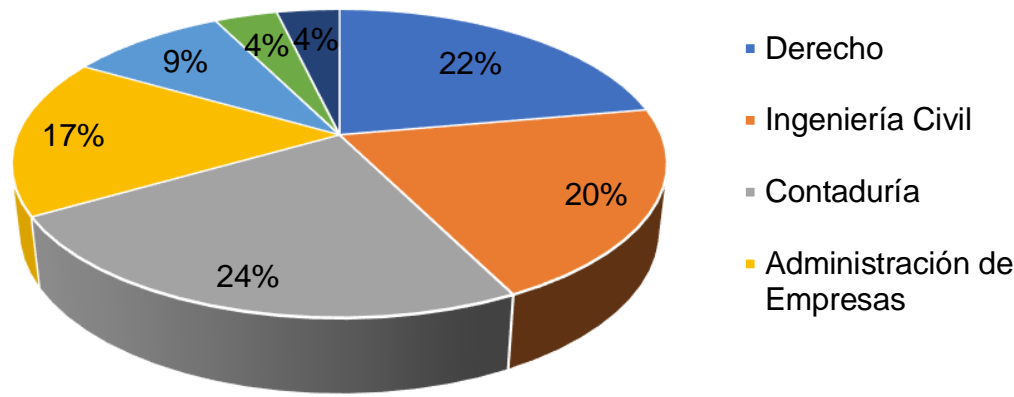

Fig. 1: Programa académico al cual están inscritos los profesores que hacen parte de la investigación

En cuanto a la edad de los profesores que hacen parte de la investigación, se colige que el $50 \%$ de los profesores que ejercen su actividad de enseñanza e investigación en la universidad cooperativa de Colombia, se ubican en el rango de edad en más de 52 años, seguido de edades entre 32-35 años y 44-47 años (13\%). Este resultado, se sustenta en la permanencia que guarda el cuerpo profesoral en la institución. Indudablemente, en la medida que las organizaciones implementan prácticas que contribuyan a la solución de las necesidades de sus trabajadores o a su satisfacción, estos adquieren una idea de compromiso. Al mismo tiempo, en la figura 2 se logra identificar que los profesores a pesar de sus edades, solo cuentan en su gran mayoría con formación de posgrado en maestría (87\%), y un número menor de pregrado (2\%).

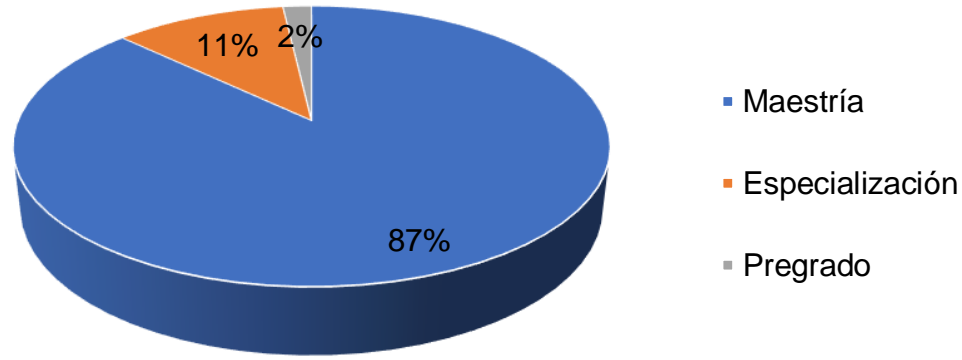

Fig. 2: Nivel educativo máximo alcanzado por los profesores que hacen parte de la investigación

La figura número 3, profundiza un poco en la percepción que tienen los profesores acerca del tema de responsabilidad social universitaria, pues dicha percepción es primordial para identificar el impacto de las políticas de RSU en la universidad estudiada, desde el enfoque de los formadores. Al observar cada una de las percepciones que manifiestan los profesores por programa académico, es importante destacar mediante un análisis cualitativo de redes las percepciones más predominantes y repetitivas que se ajustan a la pregunta ¿Qué es la RSU? Asimismo, la figura 3 reúne cuatro percepciones claves, que pueden ser explicadas a mayor profundidad desde un enfoque teórico para la presente investigación.

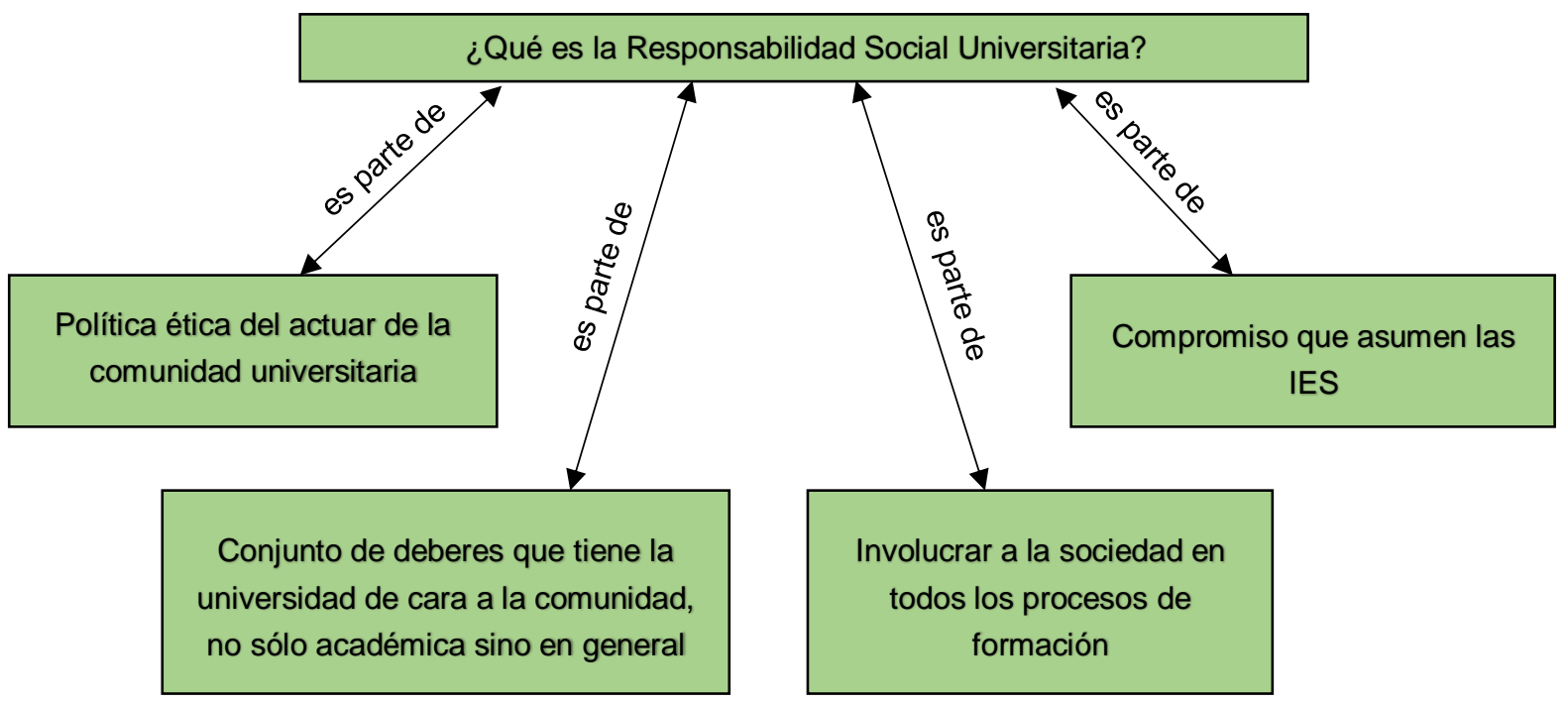

Fig. 3: Análisis de redes de la percepción de los profesores acerca de la RSU. 
En atención a las diferentes acepciones presentadas por los profesores, y a criterio de los autores, la RSU consiste en la responsabilidad que adoptan las organizaciones educativas en función de los agentes de interés; especialmente, cuando estas acciones se implementan adecuadamente y se internalizan en la organización. Así entonces, las acciones dirigidas al entorno externo previamente internalizadas tienen mayor probabilidad de ocurrencia, y pueden crear una ventaja competitiva para la institución de educación superior (Ferreira y De Oliveira, 2014). Este argumento está soportado por Flórez et al. (2017), al manifestar que no es razonable adelantar prácticas de responsabilidad social hacia afuera, cuando no se han ejercido hacia adentro. Para las universidades aplica de igual forma que la empresa tradicional; primeramente, se debe internalizar dicha práctica antes de externalizarla. En otras palabras, es inconcebible limitar el comportamiento de una organización en materia social, a realizar actividades en función de la sociedad, cuando actúa apática a las prontitudes de los grupos de interés internos (Rubio-Rodríguez et al. 2020).

\section{DISCUSIÓN}

Desde la forma como percibe el profesor el concepto responsabilidad social universitaria, indica que éste asume una posición ética en su comportamiento, con un marcado compromiso consigo mismo y con la sociedad, pero también interesado en vincular diferentes grupos sociales en los procesos de formación. Dicha posición, que puede presentarse como extraña a la luz de la conducta que axiológicamente guardar el corazón del ser humano, puede involucrar otros deseos que conducen a generar efectos contrarios. Un sinnúmero de investigaciones previas, han analizado el grado de integración de las iniciativas que presentan los agentes de interés de las organizaciones, incluidas las universidades, cuyo objetivo se ha centrado en promover un comportamiento socialmente externalizado que impacte efectivamente (Romolini et al. 2015; Beddewela et al. 2017).

No obstante, las actuaciones de quienes fungen en calidad de agentes de interés, llámese profesor, o cualquier otro actor, que propendan por mejorar las condiciones de vida del entorno externo, estriban en gran manera de los órganos de gobierno de las instituciones universitarias para incorporar este empeño de los interesados, que apelan a un modelo de gobernanza reticular y no a un modelo de estructura vertical que prevalece en algunas instituciones. Así lo revalida Wigmore-Álvarez et al. (2020), cuando sostienen que los gobiernos correspondientes responsables de la educación universitaria, consideran la manera de participar voluntariamente bajo un marco de referencia para ejercer las prácticas de responsabilidad social. Afirman además, que estas son normalmente generales, pero mediadas por pautas que regulan su cumplimento.

Respecto a la ética como política para actuar en la comunidad universitaria, la cual da cuenta a una respuesta generalizada tomada de la población encuestada, se constituye en un tema controversial, dado, como se afirmó precedentemente, obedece a la conducta del ser humando, que en ocasiones traiciona y quebranta su voluntad. Si ser exhaustivos, los estados comportamentales quedan totalmente supeditados al denuedo con el que se afronta el mundo de la vida, que en palabras de Jürgen Habermas, "se compone de la cultura, la sociedad y la personalidad. Estos elementos hacen referencia a pautas apropiadas de relaciones sociales y al modo de ser de las personas y de comportarse" (Mah, 2000, p. 14). De ahí que, An et al. (2017), descubriera que variables como el tamaño, la naturaleza pública o privada de la universidad o la presencia de cátedras de responsabilidad social, no son estadísticamente garantizadoras en transparencia ni en educación ética.

Otro de los puntos objeto de discusión, se relaciona con el compromiso que las universidades deben asumir con su entorno; respecto a ello, es vital que la universidad replantee su conducta, evalué y gestione los procesos de mejora continua que le permitan dimensionar pautas donde propiciar un ambiente que lleve a sus actores a participar en encuentros de integración con la sociedad (Astiti, 2014). Esto, se debe tener en cuenta, en vista de que el ordenamiento de la educación actual se dirige desde la base del compromiso que como individuos tenemos con el medio en el cual se gestan teorías de responsabilidad individual, idóneas para generar un sentido de responsabilidad social acorde a los diversos momentos por los que atraviesa el mundo. Así lo manifiestan Marti-Noguera et al. (2014), al indicar que "la formación de profesionales socialmente responsables en la universidad se ha convertido en una misión reconocida por la UNESCO" ( $p$. 160).

De acuerdo a lo anterior, la implicación que presenta la planeación y la implementación de las acciones en materia de RSU se relaciona con el clima organizacional que ostente la universidad. Esto, porque las empresas socialmente responsables son las que constantemente se preocupan por la dignidad humana, a través del cuidado integral de todas las actividades que directa o indirectamente recaen sobre el personal participe de la organización (Navas y González, 2016). Esto es importante para el desarrollo sano de la cultura organizacional; implementar acciones relacionadas con el salario emocional permiten satisfacer las necesidades que demandan los interesados, con lo cual, se generan oportunidades de bienestar y calidad para el personal de la organización. De lo señalado, se desprende que el salario emocional se constituye en 
un factor primordial en el desarrollo de las actividades laborales del ser humano. Sustento de ello, se presenta el estudio elaborado por Urquijo et al. (2019), en el que deducen preliminarmente que el reconocimiento a las personas es un aspecto relevante en la consecución de los logros profesionales.

En último lugar, puede existir el mayor consenso por parte de los profesores para mejorar el comportamiento de la universidad en relación a la responsabilidad social, pero, mientras la universidad no evidencie voluntad institucional, las implicaciones en la gestión de la RSU gozarán de poca representación. Así lo revalida Wigmore-Álvarez et al. (2020), cuando sostienen que los gobiernos correspondientes responsables de la educación universitaria, deben participar activamente bajo un marco de referencia para ejercer las prácticas de responsabilidad social. Afirman además, que estas son regularmente generales, pero mediadas por pautas que legalizan su cumplimento. Así las cosas, las actuaciones de quienes fungen en calidad de agentes de interés, llámese profesor o cualquier otro actor, que propendan por optimar las condiciones del entorno, dependerán en gran manera de los órganos de gobierno de las instituciones universitarias para incorporar este empeño de los interesados, que apelan a un modelo de gobernanza reticular y no a un modelo de estructura vertical que prevalece en algunas organizaciones.

\section{CONCLUSIONES}

De acuerdo al trabajo presentado y a los resultados obtenidos, se pueden plantear las siguientes conclusiones principales:

1) El grado de formación de los profesores se concentra mayormente con grado de maestría, y son en menor número quienes alcanzan estudios de doctorado. Consciente de estar a la vanguardia, la universidad objeto de estudio viene adelantado procesos de formación posgradual en su cuerpo profesoral, con miras de competir en el mercado educativo, y sostener políticas orientadas hacia la calidad educativa, soportada en la cualificación permanente. Para ello, se deben destinar recursos y posibilitar el tiempo para que los profesores logren adelantar dicha formación, lo que contribuye a la condición de permanencia de los profesores en las instituciones de educación superior. A esto se añade que, la investigación se debe involucrar en los procesos de formación, a fin de mostrar mayor interés en desempeñar su labor formadora como profesionales competentes, comprometidos con una dinámica responsable en favor de mejorar la calidad de vida de la sociedad.

2) En cuanto la edad de los profesores, la cual concentra un porcentaje por encima de los 52 años, es considerada como alta en la institución. Sin embargo, esto respalda la política de permanencia implantada por la universidad, que evidencia el compromiso de brindar acciones que vinculen formalmente y de forma regular al profesor, con lo que éste puede ver como proyecto de vida, su estancia en la universidad. Este resultado es coherente con los programas que diseñan las universidades para acoger y alinear los proyectos personales de los profesores con los de la institución, favoreciendo su estabilidad. En la medida que el profesor tome la decisión de seguir cualificándose, entregará a la institución mayores agregados en el servicio de formación a otros, así como desarrollar producción investigativa, necesaria para la gestión de recursos y de visibilidad de la universidad.

3) Finalmente, el concepto de responsabilidad social universitaria ha ido generando algunos cambios, y eso se puede apreciar en el resultado de las encuestas administradas en los profesores. Antes, este tipo de responsabilidad era percibida como mera proyección social, es decir, lo que hacia la universidad en función de lo extrínsecamente contextualizado; hoy, es asumida por el compromiso que incluye un conjunto de deberes dirigidos a la comunidad en general, y mediados por una conducta moralista. Dicho esto, los aspectos básicos que influyen en la responsabilidad social, están determinados por la conducta, entre otros, de los profesores, en tanto que, para crear una institución educativa socialmente responsable, se requiere de un proceso inacabado que demanda el compromiso de, no únicamente un cuerpo profesoral, sino, de todos los agentes de interés que la componen.

\section{AGRADECIMIENTOS}

Los autores desean agradecer a María del Pilar García Rodríguez adscrita al programa de Ciencias Sociales y de la Educación de la Universidad de Huelva, España, por su apoyo en la dirección del trabajo de tesis doctoral titulado "Análisis de los factores que inciden en el desarrollo de la Responsabilidad Social", del cual se derivó este manuscrito.

\section{REFERENCIAS}

Astiti, W. Implementasi Green Accounting Berbasis University Social Responsibility (USR) di Universitas Negeri Yogyakarta, Nominal, Barometer Riset Akuntansi dan Manajemen, 3(2), 134-149 (2014). 
An, Y., Davey, H. y Harun, H. Sustainability Reporting at a New Zealand Public University: A Longitudinal Analysis, Sustainability, 9(9), 15-29 (2017).

Appe, S., Rubaii, N., Líppez-De Castro, S. y Capobianco, S., The Concept and Context of the Engaged University in the Global South: Lessons from Latin America to Guide a Research Agenda, Journal of Higher Education Outreach and Engagement, 21(2), 7-36 (2017).

Ayala-Rodríguez, N., Barreto, I. y otros tres autores, Social Transcultural Representations About the Concept of University Social Responsibility, Studies in Higher Education, 44(2), 245-259 (2019).

Batanero, C., Estepa, A. y Godino, J. D., Análisis Exploratorio de Datos: sus Posibilidades en la Enseñanza Secundaria, Suma, 9, 25-31 (1991).

Beddewela, E., Warin, C., Hesselden, F. y Crosslet, A., Embedding Responsible Management Education-Staff, Student and Institutional Perspectives, International Journal of Management in Education, 15(2), 263-279 (2017).

Beltrán-Llavador, J., Íñigo-Bajos, E. y Mata-Segreda, A., La responsabilidad social universitaria, el reto de su construcción permanente, Revista iberoamericana de educación superior, 5(14), 3-18 (2014).

Bernal, C., Metodología de la Investigación: Administración, Economía, Humanidades y Ciencias Sociales, Editorial Pearson, Bogotá, Colombia, (2016).

Duque, P. y Cervantes-Cervantes, L. S., Responsabilidad Social Universitaria: una revisión sistemática y análisis bibliométrico, https://doi.org/10.18046/j.estger.2019.153.3389, Estudios Gerenciales, 35(153), 451-464 (2019).

Ferreira, P. y De Oliveira, E., ¿Does Corporate Social Responsibility Impact on Employee Engagement?, https://doi.org/10.1108/JWL-09-2013-0070, Journal of Workplace Learning, 26, 232-247 (2014).

Flórez, M., Rubio-Rodríguez, G., Rodríguez, M. y Cúrvelo. J., Responsabilidad Social Universitaria "una Aproximación Desde la Percepción de la Colectividad Académica”, Revista Científica Hermes, (17), 80-103 (2017).

Fonseca, I., Bernate, J., y otros tres autores, Developing Social Responsibility in University Students, In Proceedings of the 2019 11th International Conference on Education Technology and Computers, 215-218 (2019).

Fontalvo, T. y De La Hoz, E., Diseño e Implementación de un Sistema de Gestión de la Calidad ISO 9001:2015 en una Universidad Colombiana, https://dx.doi.org/10.4067/S0718-50062018000100035, Formación universitaria, 11(1), 3544 (2018).

Jie, C. T. y Huam, H. T., Predictors of Reputation Through University Social Responsibility Practices in a Malaysian Private University: The Customer's Perspective, https://doi.org/10.17576/JKMJC-2019-3503-19, Jurnal Komunikasi: Malaysian Journal of Communication, 35(3), 316-333 (2019).

Larrán, J. y Andrades, F., Analysing the Literature on University Social Responsibility: A Review of Selected Higher Education Journals, Higher Education Quarterly, 71(4), 302-319 (2017).

Mah, H., Phantasies of the Public Sphere: Rethinking the Habermas of Historians, The Journal of Modern Historié, 72(1), 153-182 (2000).

Martí-Noguera, J., Martí-Vilar, M. y Almerich, G. Responsabilidad Social Universitaria: Influencia de Valores y Empatía en la Autoatribución de Comportamientos Socialmente Responsables, Revista Latinoamericana de Psicología, 46 (3), 160 168 (2014).

Martí-Noguera, J., Calderón, A. y Fernández-Godenzi, A., La responsabilidad social universitaria en Iberoamérica: análisis de las legislaciones de Brasil, España y Perú, Revista iberoamericana de educación superior, 9(24), 107-124 (2018).

Navas, M. y González, Z., Responsabilidad social universitaria: impactos de la universidad libre, sede Cartagena, en su gestión socialmente responsable, Revista Saber, Ciencia y Libertad, 11(1), 187-196 (2016).

Obeidat, B. Y., Exploring the relationship between corporate social responsibility, employee engagement, and organizational performance: The case of Jordanian mobile telecommunication companies, International Journal of Communications, Network and System Sciences, 9(09), 361-386 (2016).

Pedraja-Rejas, L., Araneda-Guirriman, C., Rodríguez-Ponce, E. y Rodríguez-Ponce, J., Calidad en la Formación Inicial Docente: Evidencia Empírica en las Universidades CHilenas, Formación Universitaria, 5(4), 15-26 (2012).

Ramos-Monge, E., Llinás-Audet, F. J. y Barrena-Martínez, J., Drivers and barriers of University Social Responsibility: integration into strategic plans, World review of entrepreneurship, management and sustainable development, 15(1-2), 174-201 (2019).

Rodríguez, J., La Responsabilidad Social de la Empresa en el Pensamiento de los Autores Clásicos. Origen y Desarrollo, ICADE. Revista de la Facultad de Derecho, (100), 1-16 (2017).

Rubio-Rodríguez, G., Flórez, M. y otros tres autores, La Responsabilidad Social Universitaria: Caracterización de sus Principales Stakeholders, Tendencias en la Investigación Universitaria, Fondo Editorial Universitario Servando Garcés de la Universidad Politécnica Territorial de Falcón Alonso Gamero, pp 32-46, Santa Ana de Coro, Venezuela, (2020).

Romolini, A., Fissi, S. y Gori, E., Quality Disclosure in Sustainability Reporting: Evidence from Universities, Transylvanian Review of Administrative Sciences, 11(44), 196-218 (2015). 
Santos, G., Marques, C. S., Justino, E., y Mendes, L., Understanding social responsibility's influence on service quality and student satisfaction in higher education, https://doi.org/10.1016/j.jclepro.2020.120597, Journal of Cleaner Production, 256, 12-17 (2020).

Thomas, J. y Harden, A., Methods for the thematic synthesis of qualitative research in systematic reviews, https://doi.org/10.1186/1471-2288-8-45, BMC medical research methodology, 8(1), 45-57 (2008).

Turker, D., Measuring Corporate Social Responsibility: A Scale Development Study, Journal of Business Ethics, 85(4), 411-420 (2009).

Urquijo, I., Extremera, N. y Azanza, G., The Contribution of Emotional Intelligence to Career Success: Beyond Personality Traits, https://doi.org/10.3390/ijerph16234809, International Journal of Environmental Research and Public Health, 16(23), 1-13 (2019).

Vallaeys, F., Las diez falacias de la Responsabilidad Social Universitaria, http://dx.doi.org/10.19083/ridu.12.716, Revista Digital de Investigación en Docencia Universitaria, 12(1), 34-58 (2018).

Vallaeys, F. y Álvarez, J., Hacia una Definición Latinoamericana de Responsabilidad Social Universitaria. Aproximación a las Preferencias Conceptuales de los Universitarios, Educación XX1, 22(1), 93-116 (2019).

Vallaeys, F., ¿Por qué la Responsabilidad Social Empresarial no es Todavía Transformadora? Una Aclaración Filosófica, Andamios, Revista de Investigación Social, 17(42), 309-333 (2020).

Vasilescu, R., Barna, C., Epure, M. y Baicu, C., Developing university social responsibility: A model for the challenges of the new civil society, Procedia-Social and Behavioral Sciences, 2(2), 4177-4182 (2010).

Wigmore-Álvarez, A., Ruiz-Lozano, M. y Fernández-Fernández, J., Management of University Social Responsibility in Business Schools. An Exploratory Study, The International Journal of Management Education, 18(2), 1-15 (2020).

Zavala-Guirado, M., Álvarez, M. y otros tres autores, Factores Internos, Externos y Bilaterales Asociados con la Deserción en Estudiantes Universitarios, Interacciones: Revista de Avances en Psicología, 4(1), 59-69 (2018). 